\title{
Chronic Intestinal Pseudo-obstruction
}

\author{
Khalil El-Chammas, MD ${ }^{1}$ Manu R. Sood, FRCPCH, MD²,3 \\ ${ }^{1}$ Division of Pediatric Gastroenterology, Cincinnati Children's \\ Hospital Medical Center, Cincinnati, Ohio \\ 2 Division of Pediatric Gastroenterology, Hepatology and Nutrition, \\ Medical College of Wisconsin, Milwaukee, Wisconsin \\ 3 Division of Pediatric Gastroenterology, Children's hospital of \\ Address for correspondence Manu R. Sood, FRCPCH, MD, Division of \\ Pediatric Gastroenterology, Hepatology and Nutrition, Medical \\ College of Wisconsin, Milwaukee, WI 53226 \\ (e-mail: MSood@mcw.edu).
} Wisconsin, Milwaukee, Wisconsin

Clin Colon Rectal Surg 2018;31:99-107.

\begin{abstract}
Keywords

- chronic intestinal pseudo-obstruction

- bowel motility

- antroduodenal manometry

Chronic intestinal pseudo-obstruction (CIP) is defined by either continuous or intermittent symptoms of bowel obstruction in the absence of fixed lumen excluding lesion. CIP includes a heterogeneous group of disorders which result either from diseases affecting the enteric neurons and smooth muscle lining or those involving the autonomic innervation of the bowel. Symptoms associated with CIP are nonspecific, which can sometimes contribute to the delay in recognizing the condition and making the correct diagnosis. The diagnostic workup should include imaging and manometry studies and, occasionally, full-thickness bowel biopsies for histopathological examination may be required. Multidisciplinary team approach for the management of these patients is recommended, and the team members should include a gastroenterologist, surgeon, chronic pain specialist, clinical nutritionist, and a psychologist. The treatment goals should include optimizing the nutritional status and preventing or delaying the development of intestinal failure. The majority of the patients require enteral or parenteral nutrition support, and chronic pain is a common and distressing symptom. Small bowel transplantation may be required if patients develop liver complications due to parenteral nutrition, have difficult central line access, or have poor quality of life and worsening pain despite aggressive medical management.
\end{abstract}

Intestinal pseudo-obstruction refers to a heterogeneous group of disorders with a similar phenotypic presentation characterized by obstructive intestinal symptoms in the absence of a true anatomical obstruction. ${ }^{1}$ In Ogilvie's syndrome, patient develops acute intestinal pseudo-obstruction; on the other hand, if the obstructive symptoms are present for longer than 6 months, it is categorized as chronic intestinal pseudo-obstruction (CIP). This review will mainly focus on CIP and management of children with this disease. CIP was first reported in 1958 by Dudley and his colleagues when they failed to identify a cause for obstructive symptoms during exploratory laparotomy in a group of patients. It is usually diagnosed after the exclusion of a mechanical obstruction and documentation of disordered peristalsis resulting from abnormalities of the enteric neuromuscula- ture and/or its autonomic innervation. Because of the nonspecific nature of symptoms and rarity of this disorder, it is not unusual that some patients have repeated and invasive workup before the correct diagnosis is made. There are only a few experienced pediatric motility centers that offer specialized diagnostic testing such as manometry studies which can be important in establishing the diagnosis.

\section{Epidemiology}

The exact prevalence of CIP is not known; it has been estimated that less than 100 infants are born with this condition in the United States every year. This study did not account for children who may have developed CIP later in life. A Japanese nationwide survey reported a prevalence of 3.7 in 1 million
Issue Theme Pediatric and Congenital Colorectal Diseases in the Adult Patient; Guest Editor: David M. Gourlay, MD, FACS, FAAP
Copyright (c) 2018 by Thieme Medical Publishers, Inc., 333 Seventh Avenue, New York, NY 10001, USA. Tel: +1(212) 584-4662. 
individuals. $^{2}$ More than half of the patients (56.5\%) in this study developed symptoms in the neonatal period. The criteria used for the diagnosis of CIP were very stringent and so this study may have underestimated the prevalence.

\section{Etiology}

Several different classification schemes have been used to categorize pediatric patients with CIP ( - Table 1). The authors prefer to categorize CIP patients into two broad categories: congenital and acquired. Each of these groups can be further subdivided into three histological categories: neuropathies, myopathies, and mesenchymopathies, although some patients may have coexisting pathological abnormalities. A large proportion of pediatric patients are classified under the idiopathic category because no histological abnormality can be identified despite extensive testing in these patients.

Most congenital forms of CIP are sporadic with no family history of the condition. However, autosomal-dominant ${ }^{3,4}$ and recessive $\mathrm{e}^{5-8}$ inheritance of enteric neuropathies as well as $^{9-12}$ myopathies have been reported. An X-linked recessive form of neuropathic CIP has been mapped to gene locus Xq28. ${ }^{13}$ CIP is also a common manifestation of a variety of mitochondrial myopathies resulting from mutations in nu-

Table 1 Classification of chronic intestinal pseudo-obstruction

\begin{tabular}{|l|}
\hline Onset: \\
\hline Congenital \\
\hline Acquired \\
\hline Causes: \\
\hline Familial \\
\hline Sporadic \\
\hline Degenerative \\
\hline Viral \\
\hline Inflammatory \\
\hline Autoimmune \\
\hline Toxic \\
\hline Ischemia \\
\hline Areas of the gastrointestinal tract involvement: \\
\hline Entire gastrointestinal tract \\
\hline Small bowel \\
\hline Colon \\
\hline Segments of the gastrointestinal tract \\
\hline Pathology: \\
\hline Myopathy \\
\hline Neuropathy \\
\hline Combined myopathy and neuropathy \\
\hline Aganglionosis \\
\hline Hypoganglionosis \\
\hline Abnormalities of interstitial cells of Cajal \\
\hline Connective tissue disorders \\
\hline
\end{tabular}

clear or mitochondrial genes involved in mitochondrial replication and/or oxidative respiration. Mitochondrial neurogastrointestinal encephalopathy (MNGIE) is an autosomal recessive condition caused by a mutation in the thymidine phosphorylase gene, which encodes enzymes for normal mitochondrial DNA synthesis. Similarly, CIP has also been reported in association with Alpers' disease (POLG mutations) or mitochondrial encephalopathy, lactic acidosis, and stroke-like episodes syndrome (MELAS).

Megacystis-microcolon-intestinal hypoperistalsis syndrome (MMIHS) is characterized by prenatal onset of urinary bladder distension with dilated loops of bowel and is associated with a mutation of ACTG2 gene. ${ }^{14}$ This gene encodes c2 enteric actin which is an important contractile protein of enteric smooth muscle. ${ }^{15}$ Interestingly, autosomal dominant rather than autosomal recessive inheritance was reported in the majority of the patients. Phenotypic variability has been recognized and distinct complications such as prune belly syndrome and hollow visceral myopathy have been reported.

Chronic atrial and intestinal dysrhythmia (CAID) syndrome is caused by mutations in SGOL1 gene, a component of the cohesin complex. ${ }^{16}$ In addition to cardiac rhythm abnormalities, symptoms of CIP were reported in this adult cohort. Although this disorder has not been reported in children, the authors have identified this mutation in one of their patients who was diagnosed with CIP in early childhood and then died of respiratory complications not directly related to CIP.

Acquired causes of CIP include toxin exposure during critical developmental periods in utero; substances that affect neuronal migration or maturation may affect the development of the enteric plexus, leading to CIP (-Table 2). Fetal alcohol syndrome $^{17}$ as well as in utero exposure to narcotics can damage the enteric neurons, resulting in CIP. Other acquired conditions causing CIP can result from myenteric plexus neuritis secondary to persistent viral infections or autoimmune inflammatory disorders. CIP may be a rare sequela of infections such as cytomegalovirus, ${ }^{18}$ Epstein-Barr, ${ }^{19}$ and JC virus. ${ }^{20}$

\section{Pathology}

The London Classification of Gastrointestinal Neuromuscular Pathology was established by an international working group as an organizational framework for histopathologic diagnoses associated with intestinal dysmotility. ${ }^{22}$ The normal development and changes in enteric neurons and muscular lining with age are poorly understood, and this makes the interpretation of the histopathology in pediatric patients with CIP challenging. ${ }^{22}$ It is therefore not uncommon to find no recognizable abnormalities despite a thorough microscopic evaluation of full-thickness bowel biopsies in children with CIP. A standardized approach to the evaluation of enteric neuromusculature proposed by a group of experts is expected to help improve the understanding of histopathological abnormalities associated with this disorder. Because of the low diagnostic yield of full-thickness bowel biopsies in children, some pediatric motility centers in the United States do not routinely recommend full-thickness bowel biopsies in their patients. 
Table 2 Causes of secondary chronic intestinal pseudo-obstruction

\begin{tabular}{|c|}
\hline Metabolic \\
\hline Mitochondrial cytopathies \\
\hline Autoimmune \\
\hline SLE \\
\hline Scleroderma \\
\hline Dermatomyositis \\
\hline Polymyositis \\
\hline Autoimmune myositis \\
\hline Autoimmune ganglionitis \\
\hline Infectious/Postinfectious \\
\hline Chagas' disease \\
\hline Cytomegalovirus \\
\hline Herpes zoster \\
\hline Epstein-Barr virus \\
\hline Kawasaki's disease \\
\hline Post-viral neuropathy \\
\hline Endocrine \\
\hline Diabetes mellitus \\
\hline Hypoparathyroidism \\
\hline Hypothyroidism \\
\hline Oncology/Hematology \\
\hline Chemotherapy and/or bone marrow/stem cell transplant \\
\hline Pheochromocytoma \\
\hline Ganglioneuroblastoma (paraneoplastic syndrome) \\
\hline Sickle cell disease \\
\hline Muscle disorders \\
\hline Myotonic dystrophy \\
\hline Duchenne muscular dystrophy \\
\hline Toxins \\
\hline Fetal alcohol syndrome \\
\hline Drugs \\
\hline Diltiazem and nifedipine \\
\hline Cyclopentolate/phenylephrine eye drops (neonates) \\
\hline Developmental \\
\hline Delayed maturation of interstitial cells of Cajal \\
\hline Miscellaneous \\
\hline Ehlers-Danlos syndrome \\
\hline Eosinophilic gastroenteritis \\
\hline Crohn's disease \\
\hline Radiation injury \\
\hline
\end{tabular}

Abbreviation: SLE, systemic lupus erythematosus.

Source: Adapted from Connor and Di Lorenzo.

The schematic representation of the enteric nervous system and how it regulates the bowel motor function is shown in -Fig. 1 and the histopathological abnormalities of the bowel neurons and smooth muscle lining which are associated with CIP in children are summarized below (-Table 3 ).

\section{Intestinal Myopathies}

The muscularis propria of the intestinal tract is normally composed of two layers, externa (longitudinal) and interna (circular), which are oriented perpendicular to one another. There are two forms of primary muscular malformation associated with CIP, the segmental additional muscle coat and diffuse abnormality of the muscle layering. ${ }^{23,24}$ The segmental additional muscle coat has been associated with profound segmental dilatation of the colon, but small intestine involvement has not been described. The prominent separate band of smooth muscle is located internal to the muscularis interna and external to the muscularis mucosae of an otherwise normal muscularis propria. In diffuse disease of the smooth muscle lining, the abnormal muscle layering predominantly involves the small intestine. Portions of the muscularis propria retain normal bilayered architecture, but malformed regions harbor broad fascicles of smooth muscle that course obliquely or perpendicularly.

In degenerative leiomyopathy, there is a progressive loss of enteric smooth muscle and replacement with fibrous tissue. CIP symptoms are usually present, but urinary bladder involvement, age of presentation, and the inheritance pattern are very variable. ${ }^{12,25}$ Many patients may not develop symptoms until adolescence. The two consistent histopathologic alterations reported are the myocyte degeneration and intramuscular fibrosis.

Intestinal leiomyositis is a rare disease which predominantly affects children, and less than 12 patients have been reported in world literature. ${ }^{26,27}$ It is characterized by dense and diffuse lymphocytic infiltration of the muscularis propria, with limited extension into the myenteric plexus, submucosa, or lamina propria.

In addition to the aforementioned diseases, a variety of other conditions, such as bowel ischemia, drug toxicity, and autoimmune disorders, can cause secondary myopathies. ${ }^{21}$

\section{Intestinal Neuropathies}

Neuronal intranuclear inclusion disease is a rare and heterogeneous neurodegenerative disorder that affects the central and peripheral nervous systems. There is progressive ganglion cell loss and in terminal phase, hypoganglionosis is seen. The ganglion cells show degenerative features including vacuolization, nuclear pyknosis, and irregular cytoplasmic contours. Large eosinophilic inclusions in nuclei of enteric ganglion cells are the pathognomonic histopathologic finding. In addition to CIP, other clinical features include ataxia, dysautonomia, dementia, and extrapyramidal symptoms. Infantile, juvenile, and adult forms of this disease have been described. ${ }^{26}$

Aganglionosis in Hirschsprung's disease can rarely extend into varying parts of the small intestine. Rarely, segments of the bowel show normal ganglions and intervening parts are aganglionic; this is also referred to as skip segment Hirschsprung's disease. ${ }^{28}$ It is thought that neural crest cells, during craniocaudal migration, cross the mesenteric border to a more distal part of the intestine and as a result end up well ahead of the wave front, and end up colonizing an area within the aganglionic segment. 


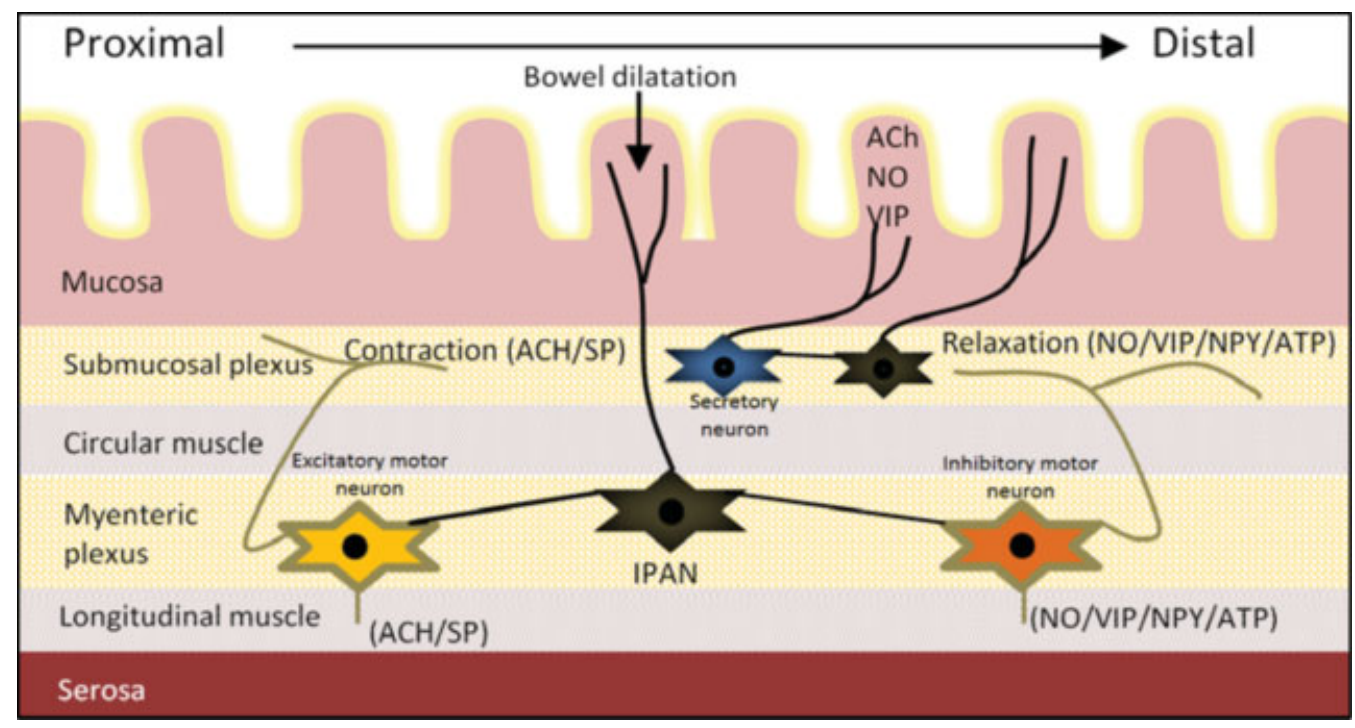

Fig. 1 Schematic representation of the enteric nervous system and connections between the neurons in the submucosal and myenteric plexus. The submucosal plexus is mainly responsible for regulating bowel secretions and blood supply. The myenteric plexus which is located between the circular and longitudinal smooth muscle layers regulates the bowel motor function. The intrinsic primary afferent neurons (IPAN) receive input from the bowel mucosa and project in both oral and anal directions. Abnormalities of the enteric neurons and smooth muscle lining are associated with chronic intestinal pseudo-obstruction. The gastrointestinal tract also receives innervation from the autonomic nervous system (not shown).

Diffuse intestinal ganglioneuromatosis is characterized by diffusely distributed transmural hamartomatous lesions which are composed of benign ganglion cells. ${ }^{26}$ It has been associated with multiple endocrine neoplasia type IIB, type I neurofibromatosis, and Cowden's disease. The hamartomatous lesions consist of mature ganglioneuromas with large ganglion cells with eccentric nuclei. Chronic constipation with megacolon is common and the pathogenesis of dysmotility is poorly understood.

Patients with intestinal hypoganglionosis present with CIP in early infancy, and the small intestine and colon show reduced number of ganglion cells per longitudinal centimeter bowel. Additionally, the plexus area and the number of ganglion cells per plexus are reduced and the ganglion distances are doubled compared with a healthy bowel. There is very low or absent activity of acetylcholinesterase in the mucosa along with significant reduction of nerve cells in the myenteric plexus and the submucous plexus ( - Table 3$)^{29}$

In inflammatory enteric ganglionitis, there is inflammation within the enteric ganglia and along the enteric nerves. Eosinophilic inflammation has been reported with eosinophilic enterocolitis, connective tissue disease, and Hirschsprung's disease. The lymphoplasmacytic inflammation can be idiopathic or associated with infections, inflammatory bowel disease, cystic fibrosis, and paraneoplastic phenomenon (e.g., neuroblastoma).

Neuronal intestinal dysplasia is a controversial histopathological abnormality, first reported by Nezelof et $\mathrm{al}^{30}$ to describe hyperplasia of the myenteric plexus and subsequently renamed by Meier-Ruge as IND (or IND type B). ${ }^{31}$ It is now considered as a morphologic phenotype affecting the submucosal plexus of the intestine, either in an isolated form or with known neuropathies such as Hirschsprung's disease or neurofibromatosis. The histological diagnostic criteria have frequently been changed in the past four decades, causing confusion and leading to some experts questioning the validity of these findings. ${ }^{22}$ The latest morphometric criteria require more than 8 neurons/ganglion (the so-called giant ganglia) in more than $20 \%$ of a minimum of 25 submucosal ganglia in patients older than 1 year. $^{22}$ These criteria were developed with 15 -mm-thick frozen sections and enzyme histochemistry. The clinical significance remains unclear because of the lack of correlation between clinical symptoms and histological findings. In some patients, the histological abnormalities improve with age.

Table 3 Newly described causes of chronic intestinal pseudoobstruction

\begin{tabular}{|l|}
\hline CAID syndrome (SGOL 1 mutation) \\
\hline $\begin{array}{l}\text { ACTG2 gene-associated megacystis-microcolon-intestinal } \\
\text { hypoperistalsis syndrome }\end{array}$ \\
\hline Mitochondrial diseases (MNGIE) \\
\hline Deficient interstitial cells of Cajal \\
\hline Viral infections (CMV, EBV, HSV, rotavirus) \\
\hline Eosinophilic enterocolitis and neuropathy \\
\hline Autoimmune (ANNA-1) \\
\hline “Idiopathic" myositis and neuropathies \\
\hline Systemic lupus erythematosus/Crohn's disease \\
\hline New drug effects-perinatal zidovudine \\
\hline
\end{tabular}

Abbreviations: CAID, chronic atrial and intestinal dysrhythmia; CMV, cytomegalovirus; EBV, Epstein-Barr virus; HSV, herpes simplex virus. 


\section{Mesenchymopathies}

Interstitial cells of Cajal (ICC) are defined by the expression of the CD117 (c-kit) protein which is a membrane receptor with tyrosine kinase activity. They are present within the submucosal, intramuscular, and intermuscular layers of the gastrointestinal (GI) tract. The myenteric ICC serve as the pacemaker and generate the bioelectric slow wave potential that leads to the contraction of the smooth muscles. Decreased number of ICCs, along with structural abnormalities, such as loss of processes and damaged intracellular cytoskeleton and organelles, has been reported in adults and children with $\mathrm{CIP}^{32}$

The tendinous collagenous tissue of the muscularis propria constitutes an integral part of the peristaltic apparatus, but the details of the collagenous tissue complex of the bowel wall are not yet fully understood. Developmental and acquired abnormalities of the bowel connective tissue have been implicated in GI motility disorders. ${ }^{33}$ Systemic sclerosis, an autoimmune disease of the connective tissue, can cause small bowel peristaltic dysfunction and CIP. ${ }^{34}$

\section{Clinical Features}

The symptoms of CIP vary from patient to patient depending on the location and extent of the GI tract involved. The most common symptoms of CIP include abdominal pain, followed by nausea and vomiting, constipation, and loose stools. Abdominal distension can worsen during exacerbation of pseudoobstruction which can be triggered by viral or bacterial infections, central line sepsis, general anesthesia, psychological stress, and malnutrition. ${ }^{35,36}$ Almost half of the children with CIP present in infancy. Antenatal ultrasound may show polyhydramnios, megacystis, and distended bowel loops. Patients with severe phenotype are usually symptomatic within the first few hours of life with remarkable bowel obstruction, while less severe cases may present months later. Forty percent of patients presenting in infancy with CIP have associated intestinal malrotation. ${ }^{1,35}$ If feeding intolerance and emesis persist after Ladd's procedure, CIP should be considered and repeated surgical interventions avoided. Adhesions are common in patients with CIP after laparotomy and can further compromise bowel propulsive function and precipitate intestinal failure.

Small intestinal bacterial overgrowth (SIBO) is a common complication and associated with worsening abdominal distention and pain. Nutritional complications due to malabsorption and micronutrition deficiencies can develop as a result of SIBO. ${ }^{35-37}$ Instead of infrequent bowel movements, patients with SIBO can develop steatorrhea.

Dilated urinary bladder and ureters are associated with CIP. Timely diagnosis can help avoid complications such as urinary tract infections and renal damage.

Because of the nonspecific nature of the symptoms associated with CIP, the condition may go unrecognized for a long time and can be confused with aerophagia, gastroparesis, functional constipation, cyclic vomiting syndrome, drug toxicity, and hypothyroidism. Sometimes, patients may be wrongly diagnosed with Munchausen-by-proxy syndrome (also known as pediatric illness falsification).

\section{Diagnosis}

To accurately diagnose CIP, imaging studies and manometry evaluation are generally required ( - Figs. 2 and $\mathbf{3}$ ). Nonspecific radiographic signs include dilated stomach, small intestine, and colon with air-fluid levels. Contrast studies help exclude an anatomical obstruction; it is crucial to plan the evacuation of barium or the use of water-soluble contrast as prolonged contrast stasis and bezoar formation are known complications of such studies in patients with CIP. Scintigraphy demonstrates delayed gastric emptying of solids or liquids. Breath hydrogen test may reveal elevation in fasting

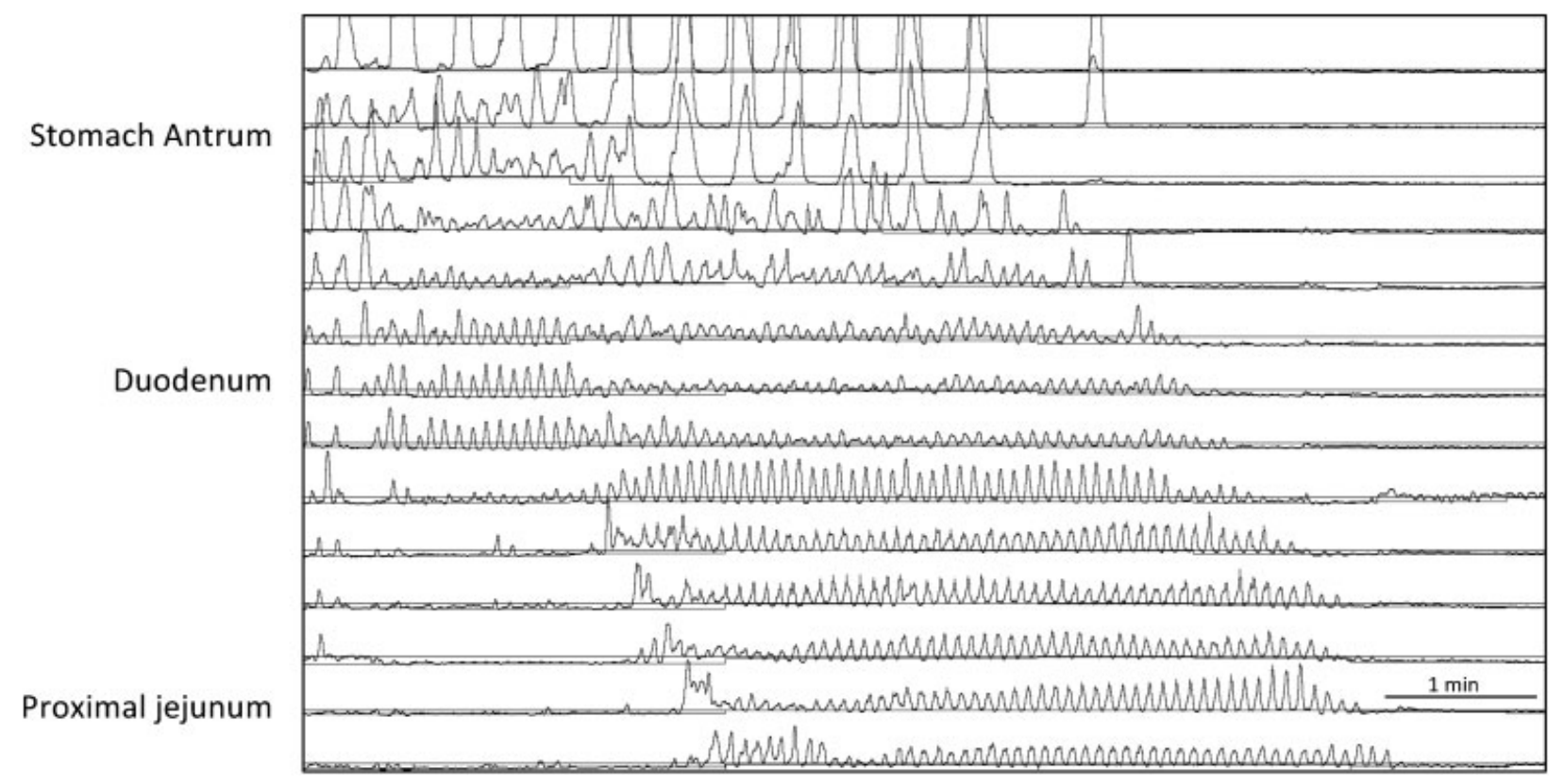

Fig. 2 Fasting antroduodenal manometry showing three per minute gastric antrum contractions and migrating motor complex in the duodenum and proximal jejunum following intravenous erythromycin. 


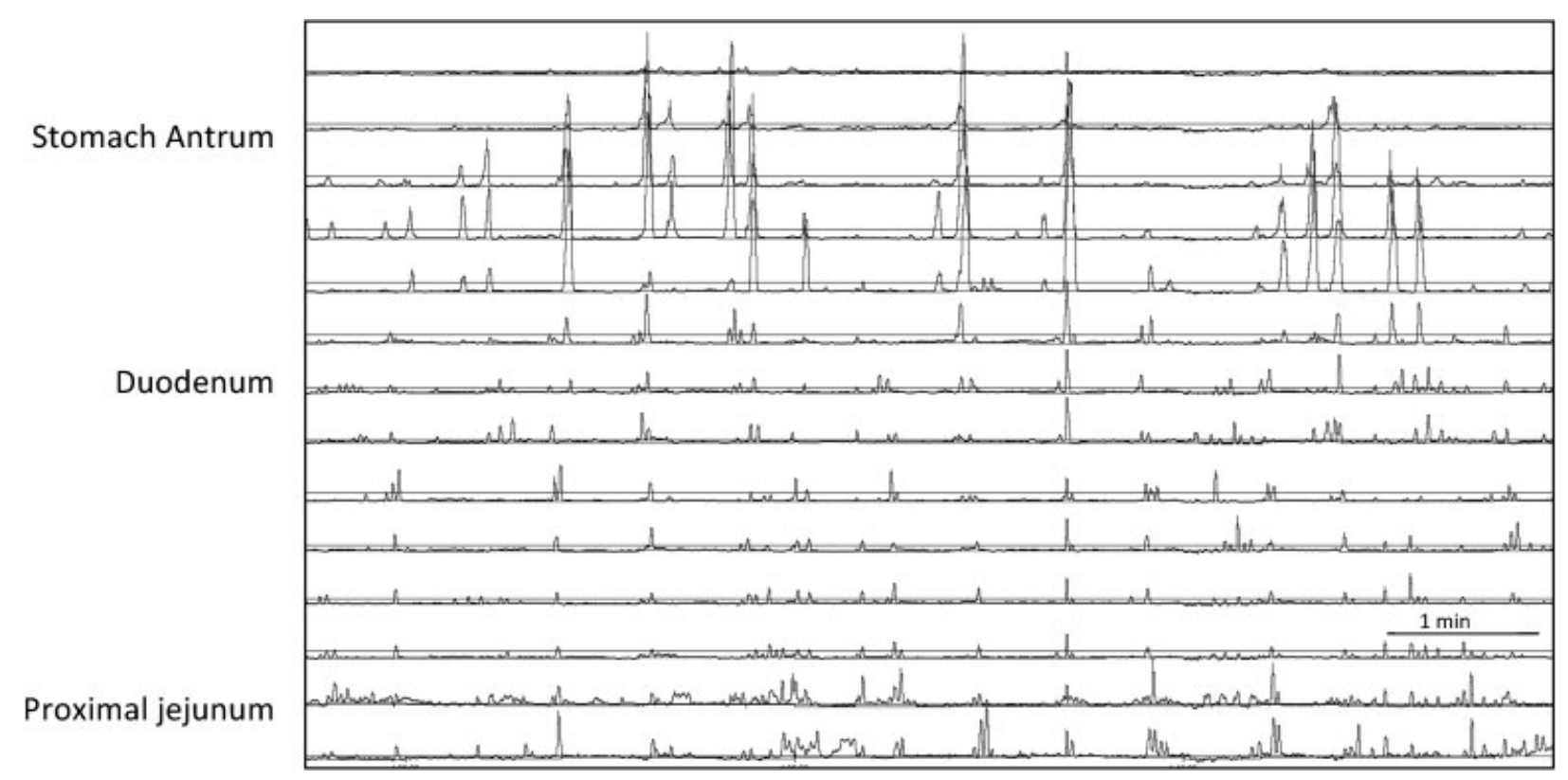

Fig. 3 Abnormal fasting antroduodenal manometry in a child with chronic intestinal pseudo-obstruction. Following intravenous erythromycin, migrating motor complex was absent, instead there was random phasic contraction and some clusters were seen in the duodenum and proximal jejunum.

breath hydrogen and rapid increase with a carbohydrate meal in the presence of SIBO.

Manometry studies are more sensitive than radiographic studies and evaluate the strength and coordination of contractions in the esophagus, gastric antrum, small intestine, colon, and anorectum. ${ }^{38,39}$ Severe abnormalities during manometry studies usually correlate with clinical symptoms. Esophageal manometry is abnormal in half of patients with CIP. Antroduodenal manometry is helpful to evaluate the stomach antrum and proximal small bowel motility. The stomach antrum normally contracts at a frequency of three contractions per minute and fasting normal small bowel contraction pattern is divided into three phases: phase I is a period of quiescence; during phase II, there are low-amplitude, random phasic contractions; and phase III/migrating motor complex (MMC) is associated with coordinated contractions lasting approximately 10 to 15 seconds (-Fig. 2). Smooth muscle disorders are characterized by low-amplitude, coordinated contractions, whereas neuropathic processes are characterized by uncoordinated contractions and the absence of MMC. ${ }^{36,40,41}$ Differentiation of CIP into neuropathic and myopathic based on manometry findings can be difficult. A normal antroduodenal manometry and absence of dilated bowel essentially exclude the diagnosis of CIP and should prompt one to consider a psychological illness. Antroduodenal manometry is abnormal in partial and complete bowel obstruction as well.

Colon manometry can show lack of gastrocolic reflex and absence of high-amplitude propagating contractions either in the entire colon or a segment of the colon. In myopathic CIP, the colon contractions are lower in amplitude and this can be difficult to differentiate from low-amplitude colon contractions associated with dilated colon. There are several pitfalls with intestinal and colon manometry studies, and movement artifact in an uncooperative and crying child can make interpretation difficult. Ideally, manometry studies should be performed when the child has no acute illness and drugs known to affect motility should be discontinued.

Full-thickness gastric antrum, small intestine, and colon biopsies are not always necessary but essential if histological evaluation of the enteric neuromusculature is needed. Most pediatric surgeons in large academic centers are able to obtain adequate tissue laparoscopically. ${ }^{42}$ It is anticipated that in the coming years, this will help improve our understanding of the histopathological abnormalities associated with childhood CIP. However, currently a vast majority of children with CIP have no identifiable abnormality of the enteric neurons or smooth muscle lining and are labeled as having idiopathic CIP. The authors' algorithm which is useful in the diagnostic workup of children with CIP is shown in - Fig. 4.

\section{Treatment}

Once the diagnosis of CIP is established, the treatment goal should be to minimize surgical intervention, preserve residual bowel motility and motor function, and optimize nutritional status. Almost one-third of patients with CIP require total or partial parenteral nutritional support and a third require total or partial enteral feeding support. ${ }^{36,37}$ Ninety percent of the deaths due to CIP are related to complications of parenteral nutrition and include central line sepsis and parenteral nutrition induced liver cirrhosis and decompensation..$^{35,38,43}$ Therefore, optimal use of enteral nutrition and discontinuation of parenteral nutrition at the earliest opportunity is important. Continuous nasogastric tube or gastrostomy feeding may be used when patients are unable to take feed orally or do not tolerate bolus feeds. Children who have gastroparesis can be fed directly into the proximal small bowel through a gastrojejunal tube or a jejunostomy can be performed. The gastrostomy can 


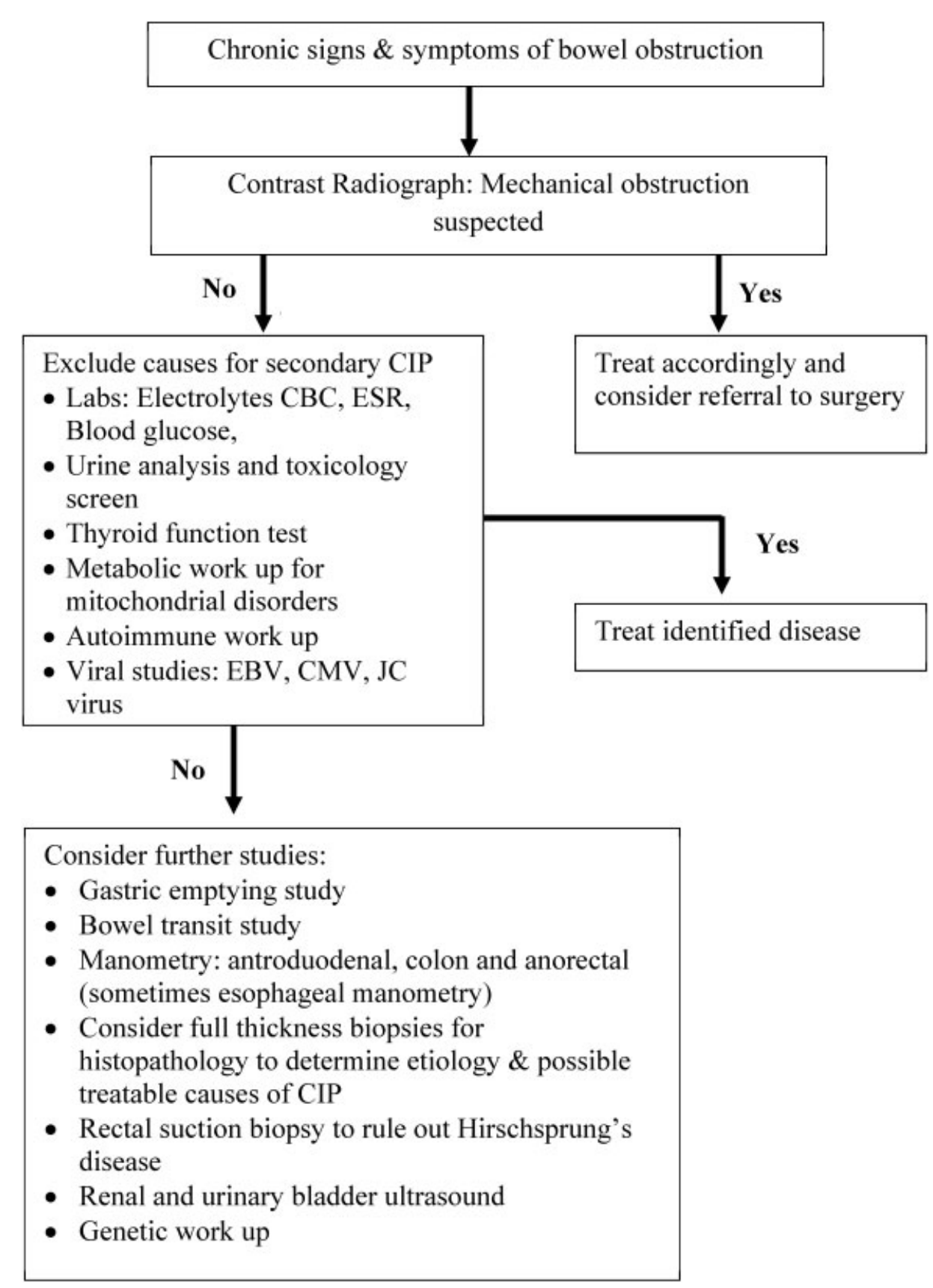

Fig. 4 Algorithm useful in the diagnostic workup of children with CIP.

also be used to vent the stomach and decompress the bowel during exacerbation of pseudo-obstruction.

Medications that stimulate intestinal motility help a minority of patients with CIP. Erythromycin (1-3 mg/kg intravenously, $3-5 \mathrm{mg} / \mathrm{kg}$ orally $)^{44}$ is a motilin receptor agonist that stimulates antral contractions (high amplitude: 3 per minute) and facilitates gastric emptying. Octreotide is a somatostatin analog that works on the small intestine and suppresses phase 2 contractions while inducing phase 3-like clustered contractions. However, these clusters may be nonpropagative or may propagate in both directions. ${ }^{45}$ Amoxicillin-clavulanic acid can increase small bowel contractions and at the same time help treat SIBO. ${ }^{46}$ Cisapride is a 5HT3 antagonist and 5HT4 agonist; it binds to the serotonin receptors in the myenteric plexus resulting in acetylcholine release and smooth muscle contraction. Cisapride has been shown to help patients with MMCs and without dilated bowels ${ }^{47}$ by increasing the number and strength of duodenal contractions. Cisapride is no longer readily available due to concerns of rare fatal cardiac arrhythmias, and its use requires an Investigational New Drug application from the Food and Drug Administration. ${ }^{48}$ Tegaserod, a 5HT4 agonist, is another drug that seemed to have potential therapeutic benefit for patients with CIP but was also withdrawn for cardiovascular concerns.

SIBO is a common complication of chronically dilated bowel and causes mucosal damage, malabsorption, fluid secretion, and gas production. ${ }^{43}$ It has been associated with steatorrhea, fat-soluble vitamin, and vitamin B12 deficiency. Judicious use of 
oral antibiotics is recommended and can help improve abdominal distension and pain. Commonly used antibiotics include amoxicillin-clavulanic acid, trimethoprim-sulfamethoxazole, and aminoglycosides. Repeated and prolonged use of oral antibiotics is associated with risk of emergence of antibioticresistant organisms as well as yeast overgrowth.

Pain is often the most distressing symptom of CIP. A gastrostomy to vent the stomach and decompress small bowel can help alleviate pain and vomiting. Chronic pain can be managed with low-dose tricyclic antidepressants and gabapentin. ${ }^{49}$ Opioids disrupt intestinal motility and should be avoided. Collaboration with a chronic pain team is usually recommended and should include mental health specialists. Other modalities for pain relief and control include cognitive behavioral therapy, hypnosis, relaxation, yoga, and massage therapy.

Constipation is initially treated with polyethylene glycol, suppositories, or enemas. Cecostomy for antegrade enemas, ${ }^{50}$ and ileostomy with/without colonic resection in carefully selected patients with colon dysmotility, may help preserve small bowel motility and delay onset of intestinal failure. If the colon manometry shows no colon contractions, ileostomy is usually the best course. ${ }^{35}$

Management of acute obstructive episodes is often challenging; the loss of fluid and electrolytes through vomiting and gastric suction can precipitate fluid and electrolyte imbalance. Careful monitoring and replacement of fluids and electrolytes is required. Unnecessary surgeries should be avoided as postoperative ileus is prolonged and development of adhesions can further compromise bowel function. Children with CIP, especially those with mega colon, are at risk of developing colon volvulus. ${ }^{51}$ Prompt diagnosis and surgical intervention can be lifesaving in this situation. Differentiating colon volvulus from pseudo-obstruction crisis can be difficult, as clinical symptoms and signs are similar. Usually patients with a volvulus will report severe abdominal pain and obstipation.

Allogeneic hematopoietic stem cell transplantation can restore thymidine phosphorylase enzyme function in patients with MNGIE and can improve clinical manifestations in the long term. Patients who have advanced liver disease associated with intestinal failure tend to have poor outcomes despite hematopoietic stem cell transplantation. ${ }^{52}$ Recently, it has been recognized that inflammatory enteric neuronal damage can be reversed by using immunosuppressive agents if diagnosed early. ${ }^{53}$

Children with poor quality of life despite appropriate medical management or those who have developed complications of parenteral nutrition or venous access problems are candidates for intestinal transplantation. ${ }^{36,54}$ The United Network for Organ Sharing data in children reported 1- and 5-year survival rates for transplantation for motility-related indications of 75 and 57\%, respectively. ${ }^{55}$ Although survival rates for intestinal transplantation have improved, they are not comparable to excellent outcome of other organ transplantation (e.g., liver and kidney). In our experience, patients who are able to attend school and have good quality of life despite needing parenteral nutrition support prefer to delay intestinal transplantation. Pediatric CIP is associated with significant morbidity and mortality usually due to the treatment these patients receive.
Although quality of life of children and their families is significantly impacted due to CIP, the long-term survival and outcome for most patients have improved over the years. ${ }^{56}$

\section{References}

1 Rudolph CD, Hyman PE, Altschuler SM, et al. Diagnosis and treatment of chronic intestinal pseudo-obstruction in children: report of consensus workshop. J Pediatr Gastroenterol Nutr 1997;24(01):102-112

2 Muto M, Matsufuji H, Tomomasa T, et al. Pediatric chronic intestinal pseudo-obstruction is a rare, serious, and intractable disease: a report of a nationwide survey in Japan. J Pediatr Surg 2014;49(12):1799-1803

3 Mayer EA, Schuffler MD, Rotter JI, Hanna P, Mogard M. Familial visceral neuropathy with autosomal dominant transmission. Gastroenterology 1986;91(06):1528-1535

4 Roy AD, Bharucha H, Nevin NC, Odling-Smee GW. Idiopathic intestinal pseudo-obstruction: a familial visceral neuropathy. Clin Genet 1980;18(04):291-297

5 Faulk DL, Anuras S, Gardner GD, Mitros FA, Summers RW, Christensen J. A familial visceral myopathy. Ann Intern Med 1978;89(5, Pt 1):600-606

6 Haltia M, Somer H, Palo J, Johnson WG. Neuronal intranuclear inclusion disease in identical twins. Ann Neurol 1984;15(04):316-321

7 Patel H, Norman MG, Perry TL, Berry KE. Multiple system atrophy with neuronal intranuclear hyaline inclusions. Report of a case and review of the literature. J Neurol Sci 1985;67(01):57-65

8 Schuffler MD, Bird TD, Sumi SM, Cook A. A familial neuronal disease presenting as intestinal pseudoobstruction. Gastroenterology 1978;75(05):889-898

9 Anuras S, Mitros FA, Nowak TV, et al. A familial visceral myopathy with external ophthalmoplegia and autosomal recessive transmission. Gastroenterology 1983;84(02):346-353

10 Ionasescu V, Thompson SH, Ionasescu R, et al. Inherited ophthalmoplegia with intestinal pseudo-obstruction. J Neurol Sci 1983; 59(02):215-228

11 Schuffler MD, Lowe MC, Bill AH. Studies of idiopathic intestinal pseudoobstruction. I. Hereditary hollow visceral myopathy: clinical and pathological studies. Gastroenterology 1977;73(02):327-338

12 Schuffler MD, Pope CE II. Studies of idiopathic intestinal pseudoobstruction. II. Hereditary hollow visceral myopathy: family studies. Gastroenterology 1977;73(02):339-344

13 Auricchio A, Brancolini V, Casari G, et al. The locus for a novel syndromic form of neuronal intestinal pseudoobstruction maps to Xq28. Am J Hum Genet 1996;58(04):743-748

14 Thorson W, Diaz-Horta O, Foster J II, et al. De novo ACTG2 mutations cause congenital distended bladder, microcolon, and intestinal hypoperistalsis. Hum Genet 2014;133(06):737-742

15 Wangler MF, Gonzaga-Jauregui C, Gambin T, et al; Baylor-Hopkins Center for Mendelian Genomics. Heterozygous de novo and inherited mutations in the smooth muscle actin (ACTG2) gene underlie megacystis-microcolon-intestinal hypoperistalsis syndrome. PLoS Genet 2014;10(03):e1004258

16 Chetaille P, Preuss C, Burkhard S, et al; FORGE Canada Consortium. Mutations in SGOL1 cause a novel cohesinopathy affecting heart and gut rhythm. Nat Genet 2014;46(11):1245-1249

17 Van Goethem G, Schwartz M, Löfgren A, Dermaut B, Van Broeckhoven C, Vissing J. Novel POLG mutations in progressive external ophthalmoplegia mimicking mitochondrial neurogastrointestinal encephalomyopathy. Eur J Hum Genet 2003;11(07):547-549

18 Sonsino E, Mouy R, Foucaud P, et al. Intestinal pseudoobstruction related to cytomegalovirus infection of myenteric plexus. N Engl J Med 1984;311(03):196-197

19 Besnard M, Faure C, Fromont-Hankard G, et al. Intestinal pseudoobstruction and acute pandysautonomia associated with EpsteinBarr virus infection. Am J Gastroenterol 2000;95(01):280-284 
20 Selgrad M, De Giorgio R, Fini L, et al. JC virus infects the enteric glia of patients with chronic idiopathic intestinal pseudo-obstruction. Gut 2009;58(01):25-32

21 Connor FL, Di Lorenzo C. Chronic intestinal pseudo-obstruction: assessment and management. Gastroenterology 2006;130(02, Suppl 1):S29-S36

22 Knowles CH, De Giorgio R, Kapur RP, et al. The London Classification of gastrointestinal neuromuscular pathology: report on behalf of the Gastro 2009 International Working Group. Gut 2010;59(07):882-887

23 Kapur RP, Correa H. Architectural malformation of the muscularis propria as a cause for intestinal pseudo-obstruction: two cases and a review of the literature. Pediatr Dev Pathol 2009;12(02):156-164

24 Smith VV, Milla PJ. Histological phenotypes of enteric smooth muscle disease causing functional intestinal obstruction in childhood. Histopathology 1997;31(02):112-122

25 Smith JA, Hauser SC, Madara JL. Hollow visceral myopathy: a lightand electron-microscopic study. Am J Surg Pathol 1982;6(03): 269-275

26 Kapur RP. Pathology of intestinal motor disorders in children. Surg Pathol Clin 2010;3(03):711-741

27 Oton E, Moreira V, Redondo C, et al. Chronic intestinal pseudoobstruction due to lymphocytic leiomyositis: is there a place for immunomodulatory therapy? Gut 2005;54(09):1343-1344

28 O'Donnell AM, Puri P. Skip segment Hirschsprung's disease: a systematic review. Pediatr Surg Int 2010;26(11):1065-1069

29 Dingemann J, Puri P. Isolated hypoganglionosis: systematic review of a rare intestinal innervation defect. Pediatr Surg Int 2010; 26(11):1111-1115

30 Nezelof C, Guy-Grand D, Thomine E. Megacolon with hyperplasia of the myenteric plexua. An anatomo-clinical entity, apropos of 3 cases [in French]. Presse Med 1970;78(34):1501-1506

31 Meier-Ruge W. Casuistic of colon disorder with symptoms of Hirschsprung's disease [author's transl]. Verh Dtsch Ges Pathol 1971;55:506-510

32 Negreanu LM, Assor P, Mateescu B, Cirstoiu C. Interstitial cells of Cajal in the gut-a gastroenterologist's point of view. World J Gastroenterol 2008;14(41):6285-6288

33 Bruhin-Feichter S, Meier-Ruge W, Martucciello G, Bruder E. Connective tissue in gut development: a key player in motility and in intestinal desmosis. Eur J Pediatr Surg 2012;22(06):445-459

34 Gyger G, Baron M. Systemic sclerosis: gastrointestinal disease and its management. Rheum Dis Clin North Am 2015;41(03):459-473

35 Heneyke S, Smith VV, Spitz L, Milla PJ. Chronic intestinal pseudoobstruction: treatment and long term follow up of 44 patients. Arch Dis Child 1999;81(01):21-27

36 Mousa H, Hyman PE, Cocjin J, Flores AF, Di Lorenzo C. Long-term outcome of congenital intestinal pseudoobstruction. Dig Dis Sci 2002;47(10):2298-2305

37 Cucchiara S, Borrelli O. Nutritional challenge in pseudo-obstruction: the bridge between motility and nutrition. J Pediatr Gastroenterol Nutr 2009;48(Suppl 2):S83-S85

38 Fell JM, Smith VV, Milla PJ. Infantile chronic idiopathic intestinal pseudo-obstruction: the role of small intestinal manometry as a diagnostic tool and prognostic indicator. Gut 1996;39(02):306-311
39 Goulet O, Jobert-Giraud A, Michel JL, et al. Chronic intestinal pseudo-obstruction syndrome in pediatric patients. Eur J Pediatr Surg 1999;9(02):83-89

40 Di Lorenzo C, Lucanto C, Flores AF, Idries S, Hyman PE. Effect of sequential erythromycin and octreotide on antroduodenal manometry. J Pediatr Gastroenterol Nutr 1999;29(03):293-296

41 Uc A, Hoon A, Di Lorenzo C, Hyman PE. Antroduodenal manometry in children with no upper gastrointestinal symptoms. Scand J Gastroenterol 1997;32(07):681-685

42 Mazziotti MV, Langer JC. Laparoscopic full-thickness intestinal biopsies in children.J Pediatr Gastroenterol Nutr 2001;33(01):54-57

43 Goulet O, Jobert-Giraud A, Michel JL, et al. Chronic intestinal pseudo-obstruction syndrome in pediatric patients. Eur J Pediatr Surg 1999;9(02):83-89

44 Di Lorenzo C, Flores AF, Tomomasa T, Hyman PE. Effect of erythromycin on antroduodenal motility in children with chronic functional gastrointestinal symptoms. Dig Dis Sci 1994;39(07):1399-1404

45 Di Lorenzo C, Lucanto C, Flores AF, Idries S, Hyman PE. Effect of octreotide on gastrointestinal motility in children with functional gastrointestinal symptoms. J Pediatr Gastroenterol Nutr 1998;27 (05):508-512

46 Gomez R, Fernandez S, Aspirot A, et al. Effect of amoxicillin/ clavulanate on gastrointestinal motility in children. J Pediatr Gastroenterol Nutr 2012;54(06):780-784

47 Di Lorenzo C, Reddy SN, Villanueva-Meyer J, Mena I, Martin S, Hyman PE. Cisapride in children with chronic intestinal pseudoobstruction. An acute, double-blind, crossover, placebo-controlled trial. Gastroenterology 1991;101(06):1564-1570

48 Raphael BP, Nurko S, Jiang H, et al. Cisapride improves enteral tolerance in pediatric short-bowel syndrome with dysmotility. J Pediatr Gastroenterol Nutr 2011;52(05):590-594

49 Rosner H, Rubin L, Kestenbaum A. Gabapentin adjunctive therapy in neuropathic pain states. Clin J Pain 1996;12(01):56-58

50 Youssef NN, Barksdale E Jr, Griffiths JM, Flores AF, Di Lorenzo C. Management of intractable constipation with antegrade enemas in neurologically intact children. J Pediatr Gastroenterol Nutr 2002;34(04):402-405

51 Altaf MA, Werlin SL, Sato TT, Rudolph CD, Sood MR. Colonic volvulus in children with intestinal motility disorders. J Pediatr Gastroenterol Nutr 2009;49(01):59-62

52 Halter JP, Michael W, Schüpbach M, et al. Allogeneic haematopoietic stem cell transplantation for mitochondrial neurogastrointestinal encephalomyopathy. Brain 2015;138(Pt 10):2847-2858

53 Cucchiara S, Borrelli O, Salvia G, et al. A normal gastrointestinal motility excludes chronic intestinal pseudoobstruction in children. Dig Dis Sci 2000;45(02):258-264

54 Bond GJ, Reyes JD. Intestinal transplantation for total/near-total aganglionosis and intestinal pseudo-obstruction. Semin Pediatr Surg 2004;13(04):286-292

55 Lao OB, Healey PJ, Perkins JD, Horslen S, Reyes JD, Goldin AB. Outcomes in children after intestinal transplant. Pediatrics 2010; 125(03):e550-e558

56 Schwankovsky L, Mousa H, Rowhani A, DI Lorenzo C, Hyman PE. Quality of life outcomes in congenital chronic intestinal pseudoobstruction. Dig Dis Sci 2002;47(09):1965-1968 